\title{
Arcobacter cryaerophilus
}

National Cancer Institute

\section{Source}

National Cancer Institute. Arcobacter cryaerophilus. NCI Thesaurus. Code C86154.

A species of Gram negative, rod shaped bacteria assigned to the phylum Proteobacteria.

This bacteria exhibits darting motility by single polar flagella, does not hydrolyze esculin, is indole and urease negative and shows susceptibility to nalidixic acid. A. cryaerophilus has been isolated from the reproductive tract, feces and milk from animals and may be pathogenic in both animals and humans. 